\title{
Learning and Memory Deficits Upon TAU Accumulation in Drosophila Mushroom Body Neurons
}

\author{
Andreas Mershin, ${ }^{1}$ Elias Pavlopoulos, ${ }^{2,3}$ Olivia Fitch, ${ }^{2}$ Brittany C. Braden, ${ }^{2}$ \\ Dimitri V. Nanopoulos, ${ }^{1,4,5}$ and Efthimios M.C. Skoulakis ${ }^{2,3,6,7}$ \\ ${ }^{1}$ Department of Physics and ${ }^{2}$ Department of Biology, Texas A\&M University, College Station, Texas 77843, USA; ${ }^{3}$ Institute \\ of Molecular Biology and Genetics, BSRC "Alexander Fleming," Vari, Greece 16672; ${ }^{4}$ Astro Particle Physics Group, Houston \\ Advanced Research Center (HARC), The Mitchell Campus, Woodlands, Texas 77381, USA; ${ }^{5}$ Academy of Athens, Chair \\ of Theoretical Physics, Division of Natural Sciences, Athens 10679, Greece
}

\begin{abstract}
Mutations in the neuronal-specific microtubule-binding protein TAU are associated with several dementias and neurodegenerative diseases. However, the effects of elevated TAU accumulation on behavioral plasticity are unknown. We report that directed expression of wild-type vertebrate and Drosophila TAU in adult mushroom body neurons, centers for olfactory learning and memory in Drosophila, strongly compromised associative olfactory learning and memory, but olfactory conditioning-relevant osmotactic and mechanosensory responses remained intact. In addition, TAU accumulation in mushroom body neurons did not result in detectable neurodegeneration or premature death. Therefore, TAU-mediated structural or functional perturbation of the microtubular cytoskeleton in mushroom body neurons is likely causal of the behavioral deficit. These results indicate that behavioral plasticity decrements may be the earliest detectable manifestations of tauopathies.
\end{abstract}

The dynamic microtubular cytoskeleton is thought to be essential for the complex structural and functional polarity in neurons (Baas 1999; Garcia and Cleveland 2001). Axonal microtubules, unlike their dendritic counterparts, appear uniformly oriented, indicating unique properties and functions (Baas 1999). In addition to the dynamic instability of anchored microtubules (Mitchison and Kirschner 1984), they may have both ends free (Garcia and Cleveland 2001), allowing subunit addition to one end and loss from the other (treadmilling; Panda et al. 1999). These properties are thought to underlie microtubule stability, length, interactive capacity, and axonal transport (Baas 1999). Microtubule stability, interactive properties, and transport are mediated at least in part by TAU, a microtubule-associated protein preferentially distributed in axons of central nervous system neurons (Buee et al. 2000).

TAU binds to microtubules through three or four imperfect highly conserved repeats located near its $C$ terminus. In vertebrates, regulated alternative splicing yields multiple TAU isoforms differing by the length of their $\mathrm{N}$ termini and the presence of three (3R) or four (4R) microtubule-binding repeats, whose ratio within neurons appears essential for their survival and function (Buee et al. 2000; Lee et al. 2001). Human mutations that alter TAU binding to microtubules, or the ratio of $3 \mathrm{R} / 4 \mathrm{R}$ isoform accumulation, result in progressive formation of intraneuronal and glial fibrillar tangles, thought to be causal of degenerative neuronal loss (Buee et al. 2000; Lee et al. 2001). Neurofibrillary tangles (NFTs), comprised mostly of aggregated TAU, characterize dementing cognitive disorders including the fronto-temporal dementias with Parkinsonism (FTDP-17), Progressive Supranuclear Palsy, and Alzheimer's disease among other such "tauopathies" (Garcia and Cleveland 2001; Lee et al. 2001). Although muta-

\footnotetext{
6Present address: Institute of Molecular Biology and Genetics, BSRC "Alexander Fleming," Vari, Greece 16672; skoulakis@fleming.gr. ${ }^{7}$ Corresponding author.

E-MAIL eskoulakis@mail.bio.tamu.edu; FAX (979) 845-6305.

Article and publication are at http://www.learnmem.org/cgi/doi/10.1101/ $\operatorname{lm} .70804$.
}

tions in the human TAU gene have been unequivocally linked to FTDP-17 (Lee et al. 2001), the exact role of TAU dysfunction in the cognitive pathology of these diseases is unknown.

The recently identified Drosophila protein (dTAU) contains four putative tubulin-binding repeats (Heidary and Fortini 2001). They exhibit $42 \%$ and $46 \%$ identity (62\% and $66 \%$ similarity) with the respective sequence of bovine (bTAU) and human (hTAU) homologs (Himmler 1989; Himmler et al. 1989; Ito et al. 1997; Heidary and Fortini 2001). Although the $\mathrm{N}$ terminus of the fly protein is highly charged, it lacks the repeats present in many vertebrate isoforms (Heidary and Fortini 2001).

Animal models of tauopathies have focused on NFT formation and neurodegeneration. Transgenic mice expressing $3 \mathrm{R}$ or 4R splice variants exhibit mild motor deficits and neuropathology without apparent NFTs (Hutton et al. 2001). In contrast, animals expressing transgenes harboring FTDP-17-linked mutations develop modest NFTs, progressive motor deficits, and neurodegeneration (Lewis et al. 2000; Hutton et al. 2001). Expression of human wild-type and FTDP-17-linked mutations in Drosophila resulted in age-dependent neurodegeneration without NFTs (Wittman et al. 2001) unless wild-type TAU was phosphorylated by overexpressed Drosophila glycogen synthase kinase-3 (Jackson et al. 2002). Interestingly, elevated nonfilamentous TAU in the "pretangle" (non-NFT) state observed in tauopathies may underlie preneurodegeneration cognitive symptoms including memory loss (Brion et al. 1999; Hutton et al. 2001). Indeed, triple-transgenic mice harboring tau, presenilin 1 (PS1), and $A \beta$ Peptide Precursor (APP) mutant transgenes, manifest synaptic dysfunction prior to development of plaques, NFTs, and overt degeneration (Oddo et al. 2003). Synaptic dysfunction likely underlies the dementia-associated deficits, but the effect of elevated pre-NFT TAU in this is unclear.

To investigate whether associative learning and memory are affected by elevated TAU, we directed expression of wild-type tau transgenes in adult Drosophila mushroom body neurons. The mushroom bodies (MBs) are bilateral neuronal clusters in the dorsal posterior cortex of each Drosophila brain lobe essential for olfactory learning and memory (Roman and Davis 2001). The 
dendrites (calyces), where inputs arrive conveying sensory information, lie ventrally to the cell bodies, while their axons fasciculate to form the pedunculus, which projects to the anterior of the brain. There, it bifurcates with processes extending medially ( $\beta$, $\beta^{\prime}$, and $\gamma$ lobes) and others projecting dorsally to comprise the $\alpha$ and $\alpha^{\prime}$ lobes (Crittenden et al. 1998). We report that accumulation of wild-type bovine, human, and Drosophila TAU in mushroom bodies precipitated a substantial decrement in associative learning and memory retrieval or stability. However, wild-type TAU accumulation did not result in neurodegeneration, early death, or decline in nonassociative processes and sensory perception.

\section{RESULTS}

\section{GAL4 Driver Expression Pattern and TAU Accumulation in Mushroom Body Neurons}

Directed accumulation of TAU in the mushroom bodies was achieved from UAS-btau, UAS-htauwt1, and UAS-dtau transgenes driven in these neurons with GAL4 (Brand and Perrimon 1993). To eliminate potential complications caused by expression of TAU in the developing nervous system, we selected strains expressing GAL4 in the mushroom bodies (MB drivers) with restricted temporal expression. We focused on MB drivers c492 and c772 because they direct preferential expression in late pupal and adult mushroom body neurons (Armstrong et al. 1998). The expression pattern of both MB drivers is shown in Figure 1. Both c492 and c772 GAL4 drivers directed preferential bTAU distribution in the dendrites (Fig. 1A,B), the fasciculated axons (Fig. 1C,D), and lobes (Fig. 1E,F) of mushroom body neurons. However, accumulation in the $\gamma$ lobes appeared reduced in c492 in comparison with the robust staining under c772 (Fig. 1E,F). In addition, c772 directed bTAU accumulation in ellipsoid body ring neurons (Fig. 1D), whereas barely above background staining was observed throughout the CNS. In addition to the mushroom bodies, c492 directed TAU accumulation in antennal lobe intrinsic neurons and neurons of the subesophageal ganglion (Fig. 1C,G). The temporally restricted expression of TAU to late pupae and adult heads was verified with Western blots (data not shown). Although accumulation of the bovine protein (bTAU) was used to monitor the expression patterns under c492 and c772 because of the availability of an anti-bTAU monoclonal antibody, identical results were obtained with a polyclonal antibody that recognizes the human isoform. A working antibody specific to the Drosophila protein was not available.

To investigate the relative level of TAU accumulation within adult heads, we performed semiquantitative Western analyses. Vertebrate TAU was present in head lysates of animals carrying both vertebrate tau transgenes and the MB drivers, but not in strains carrying either of them alone (Fig. 2). The level of bTAU did not appear to change significantly over a 3 -wk period, indicated by densitometric quantification of results from three independent experiments represented by that shown in Figure 2A. Similarly, the level of hTAU appeared relatively constant (Fig. 2B; data not shown). In addition, the results in Figure 2, A and B, indicate that transgenic protein accumulation was higher under the c772 driver. Results from RT-PCR investigation of $d t a u$ RNA accumulation from the UAS-dtau4 transgenic line were consistent with that observation (Fig. 2C). Therefore, in vertebrate and Drosophila tau-expressing transgenic animals, the overall level of TAU within the mushroom bodies is likely to be much higher than normal and represent a condition of elevated TAU accumulation in these neurons.
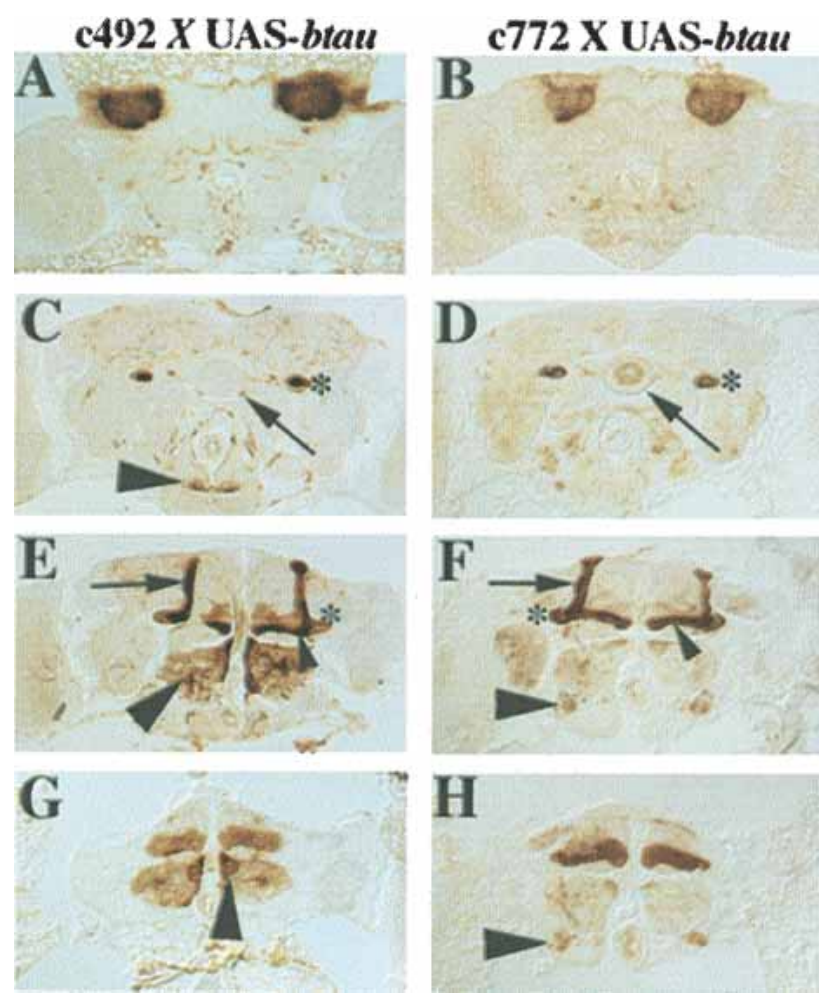

Figure 1 Expression of bTAU in mushroom body neurons. Immunohistochemical detection of bovine TAU (bTAU) accumulation within adult Drosophila brain in $5-\mu \mathrm{m}$ frontal paraffin sections challenged with a monoclonal anti-bTAU antibody. Dorsal is up in all photographs. Accumulation of bTAU is shown in c492; UAS-btau $(A, C, E, G)$, and C772; UASbtau $(B, D, F, H)$ heterozygotes. $(A, B)$ Accumulation of bTAU within the dendrites (calyxes) of mushroom body neurons in the posterior of the head. $(C, D)$ bTAU accumulates within mushroom body pedunculi (asterisks), the ellipsoid body (arrow) of c772; UAS-btau, but not of c492; UAS-btau heterozygotes. In the latter, bTAU was found within distinct neurons of the subesophageal ganglion (arrowhead). (E,F) In the axonal projections of mushroom body neurons, bTAU was abundant in the $\alpha$ (arrow) and $\beta$ (arrowhead) lobes, but in lesser amounts in the $\beta^{\prime}$ lobe and heel (asterisks) in c492; UAS-btau animals. In addition, bTAU was found in intrinsic neurons of the antennal lobe (large arrowhead). However, in c772; UAS-btau animals, the protein was found throughout the $\alpha, \alpha^{\prime}$ (arrow), and $\beta$ (arrowhead) lobes and heel (star) of the mushroom bodies and the antennal nerve (large arrowhead). $(G, H)$. In contrast to the modest accumulation of bTAU in the $\gamma$ lobes of c492; UAS-btau brains, the protein was abundant in the $\gamma$ lobes of c772; UAS-btau brains. Large arrowheads indicate expression within antennal lobe neurons for $\mathrm{C} 492$; UAS-btau and the antennal nerve for c772; UAS-btau animals.

\section{Accumulation of Wild-Type TAU in the Mushroom Bodies Does Not Affect Viability or Cause Neurodegeneration}

Pan-neural accumulation of human wild-type and mutant TAU proteins in the Drosophila nervous system from embryogenesis to adulthood, or targeted expression in cholinergic neurons has been reported to result in neurodegeneration and premature death of adult flies (Wittman et al. 2001). To determine whether TAU accumulation restricted to late pupal and adult mushroom bodies and the other brain neurons described above affects viability, we evaluated the survival of btau-, htauwt1-, and dtauexpressing flies over a period of $30 \mathrm{~d}$ posteclosion (Table 1). Because both male and female animals are typically used for our behavioral experiments, we used mixed-sex populations to evaluate survival, unlike previous studies (Buchanan and Benzer 1993; Wittman et al. 2001). We hypothesized that accumulation of 
$\mathbf{A}$
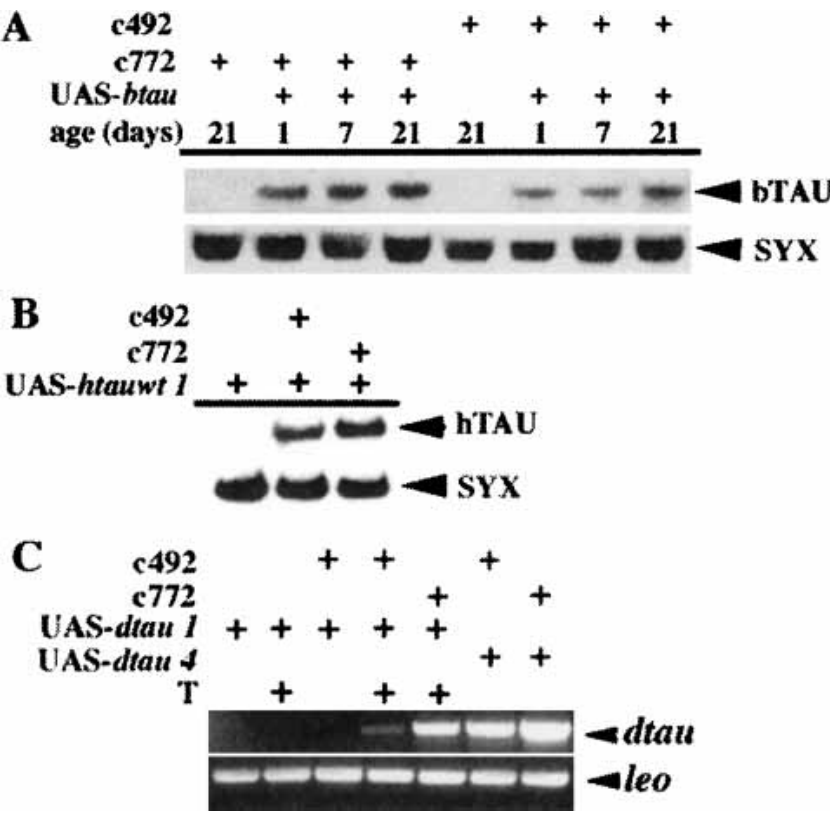

Figure 2 TAU accumulation in adult Drosophila heads. (A) A semiquantitative Western blot of $15 \mu \mathrm{g}$ from adult head lysates obtained from animals aged 7-21 d as indicated. The genotypes of the animals used are indicated above for each lane. The blot represents one of a triplicate set that was used to quantify the amount of bovine TAU (bTAU) in the lysates densitometrically using the level of syntaxin (SYX) as a reference. There was no significant change in the level of bTAU over the period tested. (B) Expression of human TAU (hTAU) in adult Drosophila heads. Here $15 \mu \mathrm{g}$ of head lysate from animals of the indicated genotypes aged 3-7 d was used. As with $A$, expression under the 4992 mushroom-body driver was more pronounced. (C) Overexpression of Drosophila tau RNA (dtau) in adult heads. One of three independent experiments is shown. The genotype of the animals is indicated above each lane. (T) Incubation for 48-52 $\mathrm{h}$ at $29^{\circ} \mathrm{C}$ prior to tissue isolation. The leo RNA was used as quality control for the RT-PCR and serves as a semiquantitative indicator of the relative levels of $d$ tau. The level of $d$ tau 1 under $\mathrm{C} 772$ at $23^{\circ}-24^{\circ} \mathrm{C}$ was investigated in a different experimental set and is not shown here, but it was found at the same or greater level than that of $\mathrm{c} 492 /+$; dtau1/+ after $48-52 \mathrm{~h}$ of incubation at $29^{\circ} \mathrm{C}$.

TAU within the mushroom bodies would not affect survival because these neurons are dispensable for viability (DeBelle and Heisenberg 1994). However, the effect of TAU accumulation on longevity in additional neurons where c772 and c492 drivers are active (Fig. 1) was unknown. Expression of vertebrate or Drosophila tau in adult mushroom body and other brain neurons did not result in decreased survival (Table 1). Because 3-6-d-old flies are typically used for behavioral experiments, we limited our systematic observations to $30 \mathrm{~d}$. A similar lack of overt differences from control strains was observed for a limited set of animals that were evaluated for viability for four additional weeks (data not shown). Furthermore, TAU accumulation did not appear to result in gross morphological differences from control strains or decreased fecundity and vigor.

Although the mushroom bodies are not essential for viability, TAU-mediated degeneration of these neurons would severely impair behavioral plasticity (DeBelle and Heisenberg 1994). To determine whether TAU accumulation in adult MBs results in degeneration, we examined their anatomy in animals that express tau transgenes using standard hematoxylin-eosin staining (data not shown) and staining with various mushroom body antigenic markers (Crittenden et al. 1998). The results obtained with the anti-LEO antibody are shown in Figure 3. Because the severity of neurodegeneration due to pan-neural tau expression increased with age and expression level (Wittman et al. 2001), we focused on 21-d-old animals to increase the sensitivity of detection. Degenerating cells and large vacuoles (Wittman et al. 2001) were not present in the mushroom bodies of controls or 21-d-old animals expressing transgenic TAU (Fig. 3A-L), indicating an absence of degeneration or inability to detect it with the methods used.

To determine whether neurodegeneration in MB neurons is detectable with our methods, we expressed the wild-type and the R406W mutation of hTAU under c492. Pan-neural expression of hTAUR406W has been reported to cause severe neurodegeneration and shorten the life span to $\sim 35 \mathrm{~d}$. Similar expression of wild-type hTAU (hTAUwt) precipitated milder degeneration and a smaller life-span reduction (Wittman et al. 2001). The 3-21-dold hTAUwt- and hTAUR406W-accumulating flies did not exhibit obvious degeneration or vacuoles in their MBs. To enhance our ability to detect degeneration in MBs, we maintained TAUexpressing animals for at least $60 \mathrm{~d}$. Mild neurodegeneration was observed in most 60-d-old hTAUwt-expressing flies, whereas severe neurodegeneration and large vacuoles were observed in the MBs of similarly aged hTAUR406W-expressing animals (Fig. 3M$\mathrm{X})$. Degeneration appeared specific to TAU-accumulating neurons, as degenerating neurons and vacuoles were observed in the MBs but not in ellipsoid body neurons where the c492 driver is inactive (Fig. 3Q,W). Interestingly, neurodegeneration was barely detectable in 60-d-old bTAU- and dTAU-expressing flies (data not shown). Therefore, MB degeneration was detectable with the methods used, but was not apparent in 3- to 21-d-old animals expressing wild-type vertebrate or Drosophila TAU. These data strongly indicate that the 3-5-d wild-type TAU-accumulating animals used in the behavioral experiments detailed below were unlikely to harbor degenerating neurons in their MBs, or that such perturbations were not detectable with the techniques used.

\section{Olfactory Learning and Memory Deficits Upon Vertebrate TAU Accumulation in Mushroom Bodies}

Because the mushroom bodies are essential for olfactory learning and memory (DeBelle and Heisenberg 1994; Roman and Davis 2001), we used negatively reinforced associative olfactory learning tasks to determine whether TAU accumulation affects these processes. Initially, we assessed the response of 3-5-d-old btauand htau-expressing animals and controls to the aversive odors used as conditioned stimuli (CS+ and CS - ) and the electric shock, the unconditioned stimulus (US). Controls and vertebrate tau-expressing animals avoided equally the aversive odors benzaldehyde and 3-octanol (CS) at two different odor concentrations given the choice of fresh air (Table 2). In addition, we tested the response of btau- and htau-expressing animals relative to controls to the attractive odor geraniol in olfactory trap assays (Philip et al. 2001). Although this odor is not task relevant, it provided an independent measure of olfactory acuity toward a qualitatively different odor. As shown in Table 2, the performance of tauexpressing animals was not significantly different from controls. Thus, btau- and htau-expressing animals retained normal olfactory responses to the odors tested. Lack of osmotactic defects was particularly important for transgenes under c492, which directs accumulation in antennal lobe neurons (Fig. 1G,H). Avoidance of electrified grids kept at $90 \mathrm{~V}$ (normal US stimulus) or $45 \mathrm{~V}$ was not different between tau-expressing animals and controls (Table 2 ), indicating a lack of mechanosensory deficits due to TAU accumulation. In addition, exposure of flies to $29^{\circ} \mathrm{C}$ for $48-52 \mathrm{~h}$ did not appear to affect their olfactory and mechanosensory responses. Collectively, these results indicate that TAU accumulation in mushroom body and other central brain neurons described above did not precipitate deficits in sensory modalities requisite for olfactory conditioning. 
Table 1. Accumulation of TAU in Central Brain Neurons Does Not Affect Viability

\begin{tabular}{|c|c|c|c|c|c|}
\hline \multirow[b]{2}{*}{ Genotype } & \multicolumn{5}{|c|}{$\%$ Survival } \\
\hline & Day 1 & Day 7 & Day 14 & Day 21 & Day 30 \\
\hline c492/+ & $100 \pm 0$ & $84.7 \pm 1.5$ & $77.9 \pm 3.1$ & $68.9 \pm 4.2$ & $64.4 \pm 3.2$ \\
\hline c772/+ & $100 \pm 0$ & $88.7 \pm 3.9$ & $76.2 \pm 2.9$ & $71.7 \pm 3.6$ & $66.1 \pm 3.6$ \\
\hline UAS-btau/+ & $100 \pm 0$ & $86.9 \pm 3.7$ & $79.5 \pm 4.1$ & $68.8 \pm 2.9$ & $62.8 \pm 4.3$ \\
\hline UAS-htauwt1/+ & $100 \pm 0$ & $83.5 \pm 3.6$ & $78.6 \pm 5.6$ & $69.5 \pm 3.6$ & $66.7 \pm 3.8$ \\
\hline UAS-dtau $1 /+$ & $100 \pm 0$ & $86.5 \pm 3.2$ & $81.9 \pm 4.3$ & $74.9 \pm 6.1$ & $63.7 \pm 4.8$ \\
\hline UAS-dtau $1 /+$ at $29^{\circ} \mathrm{C}$ & $100 \pm 0$ & $80.7 \pm 2.9$ & $71.3 \pm 3.8$ & $66.7 \pm 4.6$ & $61.9 \pm 5.3$ \\
\hline UAS-dtau $4 /+$ & $100 \pm 0$ & $83.4 \pm 3.5$ & $78.8 \pm 2.7$ & $70.3 \pm 3.1$ & $63.4 \pm 3.7$ \\
\hline c492/+; UAS-btau/+ & $100 \pm 0$ & $87.8 \pm 4.3$ & $78.1 \pm 3.1$ & $71.2 \pm 4.9$ & $65.4 \pm 4.2$ \\
\hline c492/+; UAS-htauwt1/+ & $100 \pm 0$ & $84.6 \pm 3.2$ & $78.6 \pm 4.3$ & $72.5 \pm 3.8$ & $64.5 \pm 3.9$ \\
\hline c492/+; UAS-dtau1/+ & $100 \pm 0$ & $84.8 \pm 3.4$ & $77.6 \pm 4.9$ & $72.3 \pm 4.3$ & $66.2 \pm 3.7$ \\
\hline $\mathrm{c} 492 /+; \mathrm{UAS}-d \operatorname{tau} 1 /+$ at $29^{\circ} \mathrm{C}$ & $100 \pm 0$ & $83.7 \pm 4.2$ & $73.7 \pm 3.7$ & $67.2 \pm 3.9$ & $59.5 \pm 4.7$ \\
\hline c492/+; UAS-dtau4/+ & $100 \pm 0$ & $86.2 \pm 4.2$ & $78.1 \pm 3.1$ & $73.6 \pm 3.5$ & $66.3 \pm 2.4$ \\
\hline c772/+; UAS-btau/+ & $100 \pm 0$ & $87.1 \pm 3.5$ & $77.4 \pm 3.3$ & $69.7 \pm 3.9$ & $63.2 \pm 3.3$ \\
\hline c772/+; UAS-htauwt1/+ & $100 \pm 0$ & $83.9 \pm 4.6$ & $78.8 \pm 3.7$ & $72.7 \pm 4.7$ & $67.6 \pm 5.1$ \\
\hline c772/+; UAS-dtau $1 /+$ & $100 \pm 0$ & $89.7 \pm 3.9$ & $83.6 \pm 3.8$ & $73.7 \pm 2.8$ & $64.8 \pm 3.9$ \\
\hline $\mathrm{c} 772 /+; \mathrm{UAS}-d \operatorname{tau} 1 /+$ at $29^{\circ} \mathrm{C}$ & $100 \pm 0$ & $80.3 \pm 3.6$ & $72.8 \pm 4.2$ & $62.7 \pm 4.6$ & $58.6 \pm 5.4$ \\
\hline c772/+; UAS-dtau4/+ & $100 \pm 0$ & $87.6 \pm 3.2$ & $80.2 \pm 2.9$ & $76.9 \pm 2.4$ & $67.1 \pm 4.4$ \\
\hline
\end{tabular}

An additional olfactory conditioning-relevant control is the reported (Preat 1998) decrement in response to the CS - after experiencing the coupled CS+/US stimuli. Because defects in this experience-dependent nonassociative response may underlie the associative learning and memory defects of certain mutants (Preat 1998), we quantified the reduction in CS - response following CS+/US pre-exposure. Although CS+/US pre-exposure reduced significantly subsequent avoidance of the CS -, tauexpressing animals and controls exhibited equal decrements (Fig. $4 \mathrm{~A})$. Therefore, vertebrate TAU accumulation did not cause differential responses to odor-shock pre-exposure.

To determine whether TAU accumulation in the mushroom bodies affected associative processes, we trained btau- and htauexpressing animals and controls in the LONG version of a negatively reinforced, olfactory associative learning task (Tully and Quinn 1985; Skoulakis and Davis 1996). c492/+; UAS-btauI/+, c772/+; UAS-btauI/+, and c772/+; UAS-htauwt1/+ heterozygotes exhibited a highly significant 25\%-30\% impairment in learning compared with controls (Fig. 4B, immediate). In addition, memory of the conditioned association 90 min later was significantly depressed in tau-expressing animals, and to a lesser degree at $180 \mathrm{~min}$ (Fig. 5B). These results demonstrated that TAU accumulation in the mushroom bodies compromised behavioral plasticity underlying associative olfactory learning and memory.

To examine the learning and memory deficits of btau- and htau-expressing animals more closely, we used the SHORT variant of associative olfactory training (Beck et al. 2000). Because the LONG paradigm uses a 60 -sec CS+ presentation concurrent with $1190-\mathrm{V}$ electric shocks, performance represents learning from multiple rounds of massed CS/US pairings. In contrast, for SHORT program training, a $10-\mathrm{sec}$ CS+ presentation is coupled to a single $90-\mathrm{V}$ shock, allowing assessment of learning after a single CS/US pairing (Beck et al. 2000; Cheng et al. 2001). Furthermore, performance in SHORT training improves upon multiple pairings with a 15-min intertrial interval (Beck et al. 2000; Cheng et al. 2001). This allows experimental manipulation to produce equivalent learning in control and experimental animals, a necessary condition to investigate memory stability and retrieval properties.
The results in Figure 4C demonstrate that a single CS/US pairing in btau- and htau-expressing animals yielded learning scores nearly $50 \%$ lower than those of controls. As with controls, the performance of tau-expressing animals improved upon multiple CS/US pairings. However, three CS/US pairings were necessary for tau-expressing animals to perform equivalently to controls after two such training episodes (Fig. 4C). This indicated that TAU accumulation either impaired learning elicited by each CS/US pairing, or compromised memory stability, retrieval, or a combination thereof. To distinguish between these possibilities, we trained c772; UAS-btau and c772; UAS-htauwt1 heterozygotes to the same performance level as controls (three pairings for tauexpressing animals and two for controls) and measured retention of the association after $30 \mathrm{~min}$. The tau-expressing animals exhibited a significant decrease in 30-min memory, despite performing equivalently to controls immediately after training, indicating that memory retrieval and /or stability were compromised in TAU-expressing animals. This result implies that accumulation of TAU protein within mushroom body neurons inhibits mechanisms that underlie memory stability and/or retrieval. Given the microtubule-binding properties of TAU, the results indicate that the behavioral deficits were likely precipitated by burdening these neuronal cytoskeletal elements with excessive TAU protein.

\section{Accumulation of dTAU in Mushroom Body Neurons Impairs Olfactory Learning and Memory}

Despite the apparent ability to bind Drosophila microtubules, the effects of vertebrate TAU accumulation in the mushroom bodies could result from the potential structural and conformational differences between the vertebrate TAU proteins and the native dTAU. Alternatively, the learning and memory deficits could result from the elevation of TAU within mushroom body neurons alone and the subsequent compromise in their physiology. To address these hypotheses, we generated dtau transgenic flies and investigated the effects of increasing the amount of dTAU on mushroom-body-mediated learning and memory.

We used two different $d$ tau transgenic lines that yield quantitatively very different levels of transgenic products (Fig. 2C). 


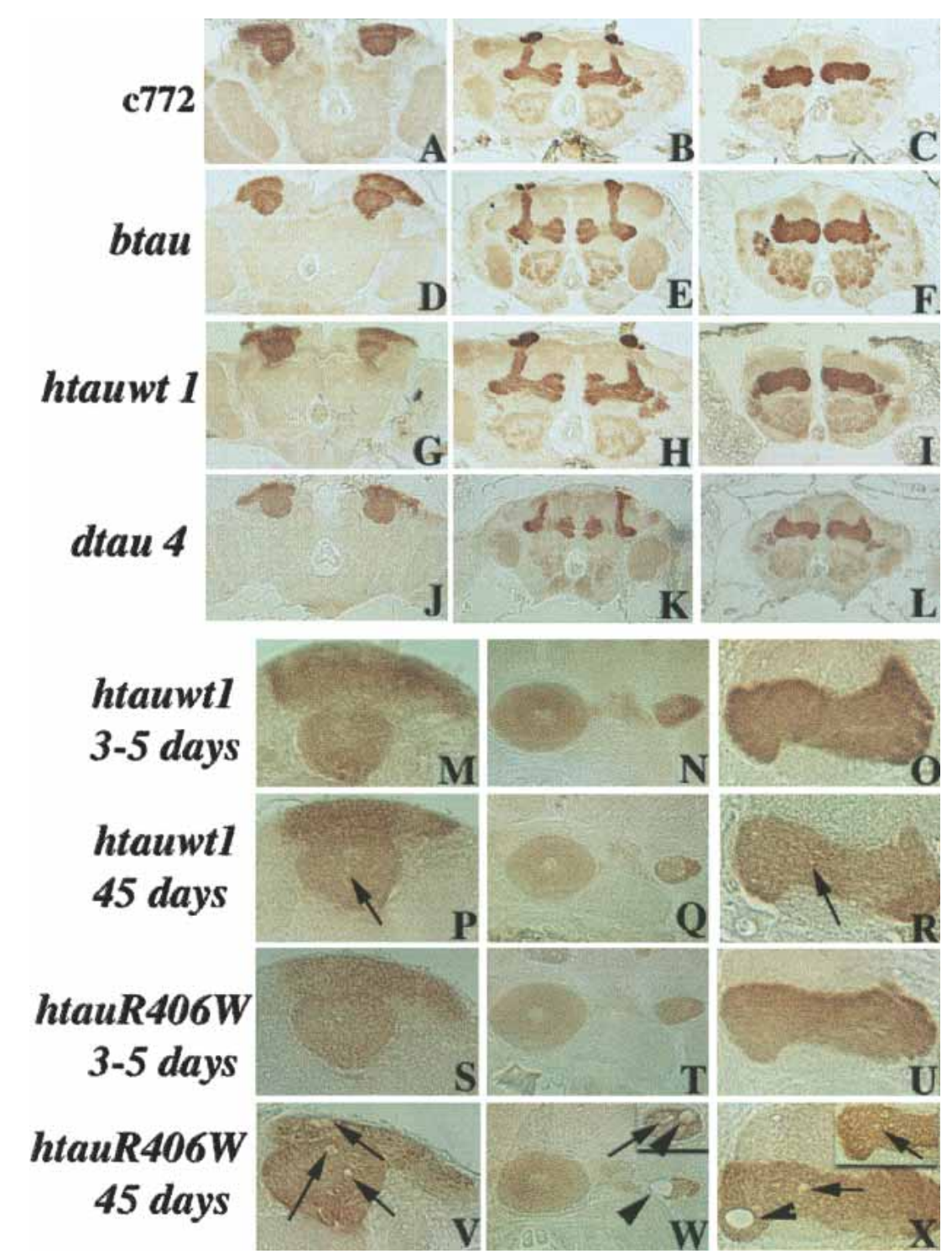

Figure 3 Neurodegeneration caused by accumulation of wild-type and mutant TAU. Degeneration of TAU-expressing neurons was investigated in 5 - $\mu \mathrm{m}$ frontal paraffin sections of the brains of TAU-accumulating animals and controls using multiple antigenic markers. In the figure, the structure of mushroom body neurons was investigated in 21-d-old animals expressing wild-type bovine $(D-F)$, human (G-I), and Drosophila $(I-L)$ tau transgenes and controls $(A-C)$ with the anti-LEO antibody that decorates most mushroom body neurons. $(M-X)$ High-magnification images from the brains of 3-5-d and 45-d-old htauwt 1 and the R406W mutation-expressing animals decorated with the anti-LEO antibody. Arrows point to degenerating neurons and arrowheads to vacuoles. Images of the affected tissues collected from independent animals have been inserted in the upper right corner in $W$ and $X$. $(A, D, J, M, P, S, V)$ Posterior sections at the level of the dendrites (calyces). ( $B, E, K)$ Anterior sections at the level of the $\alpha, \alpha^{\prime}, \beta$, and $\beta^{\prime}$ lobes and heel. $(C, F, I, L, O, R, U, X)$ Anterior sections at the level of the $\gamma$ lobe. $(N, Q, T, W)$ Sections in the middle of the head at the level of the ellipsoid body and pedunculus.

Interestingly, the level of transgenic dtau RNA in line UAS-dtau1 was undetectable when driven by c492 (Fig. 2C) and very low under c772 in heads of flies raised at $23^{\circ}-24^{\circ} \mathrm{C}$. However, the amount of RNA increased significantly if the flies were incubated at $29^{\circ} \mathrm{C}$ for $48-52 \mathrm{~h}$ prior to RNA isolation (Fig. 2C). This is likely the result of suboptimal expression caused by the chromosomal location of the transgene (position effects) in the line dtau1. The apparent increase in dtau RNA upon temperature elevation is a property of the UAS promoter and has been described previously (Duffy 2002). In wild-type animals, dTAU is distributed throughout the adult nervous system including the mushroom bodies (Heidary and Fortini 2001; E.M.C. Skoulakis, unpubl.). Accumulation of dTAU in the adult mushroom bodies did not result in a reduction in responses to sensory stimuli (Table 2), or alter re- sponses to CS+/US pre-exposure (Fig. 5A) of animals highly expressing dtau (dtau4 transgenics), indicating that as with vertebrate TAU-expressing animals, task relevant experiencedependent behaviors remain unaffected. Similar results were obtained with the low expressing dtau1 transgenics (data not shown). In contrast, accumulation of dTAU in MBs resulted in a $25 \%-30 \%$ decrease in olfactory learning and 90-min memory (Fig. 5B), similar to that observed in btau- and htauwt1-expressing animals. These results strongly implied that the decrements in learning and memory observed in btau- and htau-expressing animals were not caused by accumulation of a vertebrate protein, but rather by excess TAU within these neurons. This conclusion was further supported by investigating learning in dtau1 transgenics that express the transgene at detectable levels only after a 
Table 2. Task-Relevant Sensory Behaviors and Osmotactic Response to an Attractive Odor

\begin{tabular}{|c|c|c|c|c|c|c|c|}
\hline \multirow[b]{2}{*}{ Genotype } & \multicolumn{2}{|c|}{ Benzaldehyde $^{a}$} & \multicolumn{2}{|c|}{ Octanol $^{b}$} & \multicolumn{2}{|c|}{ Electroshock ${ }^{c}$} & \multirow[b]{2}{*}{ Geraniol $^{\mathrm{d}}$} \\
\hline & $1 \times$ & $0.1 \times$ & $1 \times$ & $0.1 \times$ & $90 \mathrm{~V}$ & $45 \mathrm{~V}$ & \\
\hline c492 & $73.24 \pm 2.31$ & $61.59 \pm 4.18$ & $78.91 \pm 4.54$ & $59.86 \pm 5.07$ & $76.31 \pm 3.61$ & $56.41 \pm 6.44$ & $68.05 \pm 4.75$ \\
\hline c772 & $.98 \pm 3.33$ & $66.57 \pm 3.79$ & $91 \pm 2.21$ & $71.23 \pm 4.54$ & $77.09 \pm 4.02$ & $60.17 \pm 5.06$ & $62.61 \pm 7.57$ \\
\hline UAS-btaul+ & $80.13 \pm 2.39$ & $59.22 \pm 2.43$ & $83.96 \pm 2.33$ & $59.04 \pm 4.55$ & $69.05 \pm 5.09$ & $58.51 \pm 4.01$ & $78.94 \pm 4.48$ \\
\hline c492/+; UAS-btau/+ & $86.31 \pm 4.33$ & $73.83 \pm 2.16$ & $87.35 \pm 4.87$ & $69.97 \pm 4.41$ & $82.91 \pm 4.09$ & $58.26 \pm 3.28$ & $67.87 \pm 4.35$ \\
\hline c772/+; UAS-btau/+ & $76.03 \pm 5.26$ & $67.55 \pm 2.73$ & $82.64 \pm 2.78$ & $74.09 \pm 6.73$ & $70.24 \pm 5.29$ & $49.11 \pm 8.41$ & $70.19 \pm 4.13$ \\
\hline UAS-htauwt 1 & $82.89 \pm 2.86$ & $68.73 \pm 3.45$ & $79.78 \pm 3.27$ & $62.37 \pm 3.87$ & $74.59 \pm 4.68$ & $56.78 \pm 5.27$ & $66.72 \pm 4.62$ \\
\hline c492/+; UAS-htauwt1/+ & $81.26 \pm 3.67$ & $64.58 \pm 2.96$ & $82.47 \pm 3.98$ & $67.79 \pm 4.39$ & $76.46 \pm 4.89$ & $54.89 \pm 4.78$ & $71.87 \pm 4.64$ \\
\hline c772/+; UAS-htauwt1/+ & $78.76 \pm 2.97$ & $67.81 \pm 3.66$ & $81.78 \pm 4.27$ & $70.91 \pm 2.98$ & $78.68 \pm 3.95$ & $53.97 \pm 5.17$ & $68.72 \pm 4.49$ \\
\hline UAS-dtau1/+ & $81.66 \pm 2.78$ & $67.12 \pm 3.26$ & $85.48 \pm 3.08$ & $66.27 \pm 3.74$ & $76.82 \pm 2.99$ & $49.93 \pm 5.34$ & $64.48 \pm 4.32$ \\
\hline UAS-dtau $1 /+$ at $29^{\circ} \mathrm{C}$ & $80.03 \pm 3.10$ & $63.93 \pm 2.93$ & $86.69 \pm 3.34$ & $61.94 \pm 3.92$ & $73.27 \pm 3.46$ & $52.78 \pm 4.97$ & $68.72 \pm 4.49$ \\
\hline UAS-dtau4/+ & $82.14 \pm 2.37$ & $63.48 \pm 3.57$ & $80.96 \pm 2.87$ & $64.36 \pm 4.28$ & $74.08 \pm 4.29$ & $56.82 \pm 4.39$ & $67.24 \pm 5.27$ \\
\hline c492/+; UAS-dtau1/+ & $77.73 \pm 3.76$ & $65.28 \pm 2.84$ & $80.46 \pm 3.24$ & $66.15 \pm 3.94$ & $79.12 \pm 4.53$ & $51.32 \pm 4.88$ & $69.47 \pm 4.78$ \\
\hline $\mathrm{c} 492 /+$ U UAS-dtau $1 /+$ at $29^{\circ} \mathrm{C}$ & $84.23 \pm 4.24$ & $71.17 \pm 4.21$ & $84.25 \pm 2.82$ & $60.82 \pm 4.08$ & $75.14 \pm 4.28$ & $52.36 \pm 4.67$ & $66.86 \pm 4.38$ \\
\hline c772/+; UAS-dtau1/+ & $78.76 \pm 2.97$ & $64.59 \pm 3.49$ & $82.47 \pm 3.54$ & $65.38 \pm 4.73$ & $75.27 \pm 3.77$ & $56.23 \pm 5.43$ & $65.84 \pm 5.49$ \\
\hline $\mathrm{c} 772 /+;$ UAS-dtau $1 /+$ at $29^{\circ} \mathrm{C}$ & $81.57 \pm 3.17$ & $69.22 \pm 3.62$ & $86.18 \pm 3.21$ & $68.17 \pm 4.46$ & $71.89 \pm 4.95$ & $55.62 \pm 4.71$ & $67.48 \pm 4.72$ \\
\hline c492/+; UAS-dtau4/+ & $84.76 \pm 2.72$ & $70.88 \pm 4.04$ & $83.85 \pm 2.73$ & $63.36 \pm 3.92$ & $76.36 \pm 4.16$ & $52.88 \pm 4.64$ & $63.87 \pm 4.97$ \\
\hline c772/+; UAS-dtau4/+ & $80.69 \pm 3.18$ & $66.47 \pm 3.29$ & $82.94 \pm 2.58$ & $67.11 \pm 4.24$ & $74.83 \pm 3.78$ & $57.74 \pm 4.89$ & $65.47 \pm 4.28$ \\
\hline
\end{tabular}

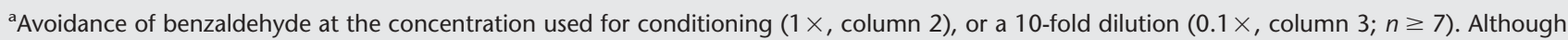
the effect of benzaldehyde dilution was significant $\left(F_{(1,271)}=9.075, p<0.005\right)$, ANOVA did not reveal differences among strains at either dilution ${ }^{\mathrm{b}}$ Avoidance of octanol as used for conditioning $(1 \times$, column 4$)$, or a 10 -fold dilution $(0.1 \times$, column $5 ; n \geq 7)$. Significant effects were revealed for odorant dilution $\left(F_{(1,267)}=11.624, p<0.005\right)$ and marginally among strains for $0.1 \times$ octanol avoidance $\left(F_{1,134)}=3.987, p<0.05\right)$. Subsequent pairwise comparisons revealed differences among all strains and c772/+, c772/+; UAS-btau/+; heterozygotes, which is unlikely to have contributed to defects in associative behaviors (Fig. 4) because the c772 control is normal in that assay.

'Avoidance of electrified grids kept at $90 \mathrm{~V}$ (column 6 ) or $45 \mathrm{~V}$ (column $7 ; n \geq 7$ ). Two-way ANOVA revealed significant effects of stimulus strength $\left(F_{1,262)}=11.873, p<0.005\right)$, but among strains either at 90 or $45 \mathrm{~V}$.

${ }^{\mathrm{d} O}$ 'smotaxis and navigation toward an attractive odor (column 8 ). There was no statistical difference in attraction toward $0.05 \%$ geraniol and entrance into the olfactory traps $(n \geq 12)$.

48-52-h period at $29^{\circ} \mathrm{C}$ (Fig. 2C). Under conditions of low dtau transcription, the performance of c492/+; UAS-dtau1/+ animals was not affected. However, elevation of dtau transcription precipitated learning deficits similar to those observed with vertebrate tau and dtau4 transgenics (Fig. 5C). Similar deficits were observed with c772/+; UAS-dtau1/+ animals (Fig. 5C), except the effect of elevating transgene expression was not as pronounced because the $\mathrm{c} 772$ driver appeared more active than $\mathrm{c} 492$ at $23^{\circ}$ $24^{\circ} \mathrm{C}$ (Fig. 2C). The effects of dTAU accumulation in the mushroom bodies were extinguished if c492/+; UAS-dtau1/+ animals were allowed a $48-52$-h rest at $18^{\circ}-20^{\circ} \mathrm{C}$ following the $48-52$-h period at $29^{\circ} \mathrm{C}$ (data not shown). These results strongly indicate that the associative learning and memory deficits in vertebrate tau- and dtau-expressing animals were precipitated by elevated TAU accumulation within mushroom body neurons and not because of the conformational differences between vertebrate and Drosophila proteins.

\section{DISCUSSION}

Directed expression of vertebrate and Drosophila tau transgenes in adult mushroom bodies appears to represent a condition of overall elevated TAU protein similar to that described in humans and animal models (Bancher et al. 1989; Braak et al. 1994; Brion et al. 1999) prior to tangle formation, and, therefore, it is suitable to investigate its effects on behavioral plasticity. Accumulation of mutant human TAU in the Drosophila nervous system from early embryos to adults resulted in neurodegeneration and severely reduced life span, which was much less pronounced if transgenes bearing the wild-type sequence were used. In contrast, directed expression within Drosophila cholinergic neurons, which are particularly sensitive to tauopathy-mediated neurodegeneration in humans and flies, did not reduce life span despite marked degeneration of these neurons (Wittman et al. 2001). In agreement with the latter and the dispensability of MBs for viability (DeBelle and Heisenberg 1994), accumulation of wild-type vertebrate or Drosophila TAU in the mushroom bodies did not appear to cause life-span reduction or overt neurodegeneration, except in very old animals. The life span was not reduced even for $h T A U R 406 \mathrm{~W}$ expressing animals exhibiting severe neurodegeneration. Moreover, the onset of neurodegeneration was significantly delayed in c772 or c492 compared with elav-GAL4-driven hTAUR $406 \mathrm{~W}$ transgenics (E.M.C. Skoulakis and S. Kosmidis, unpubl.), likely the result of using the late pupal and adult onset drivers c492 and c772.

TAU-mediated degeneration is not a likely explanation for the learning and memory decrements in the young animals used in this study for two reasons. First, the flies used for behavioral assays were younger than $7 \mathrm{~d}$, a time when degeneration was not detectable under the late pupal and adult c492 and c772 GAL4 drivers, or even the early onset elav-GAL4 driver (Wittman et al. 2001). In addition, degeneration could not have escaped detection because our methods were sensitive enough to detect it. Second, sensory and nonassociative experience-dependent behaviors necessary for associative learning were normal in tauexpressing animals. This is especially important for neurons within the antennal lobe (in tau-expressing animals under c492), whose functions appear intact in two different experienceindependent olfactory tasks (Table 1 ) and experience-dependent tasks (Figs. 4A and 5A). Degenerating antennal lobe neurons would likely precipitate olfactory deficits that were not observed in 3-7-d-old (Table 2) and 18-21-d-old animals (data not shown).

In agreement with genetic and pharmacological disruption of MB neurons (DeBelle and Heisenberg 1994; Connoly et al. 1996), TAU accumulation did not affect responses to odors (CS) or shock (US; Table 2). However, because for olfactory conditioning, normal perception of the control odor (CS - ) following ex- 
A

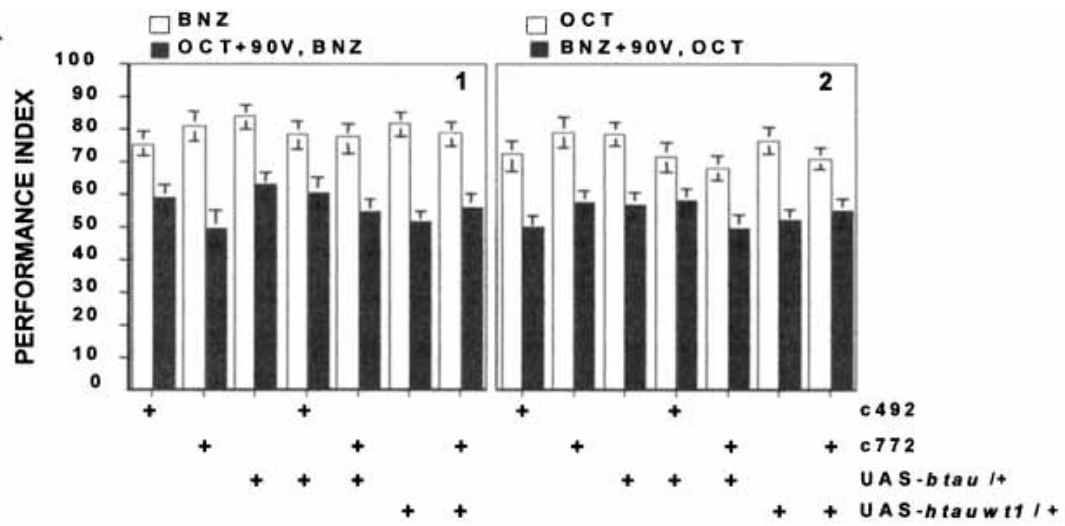

B

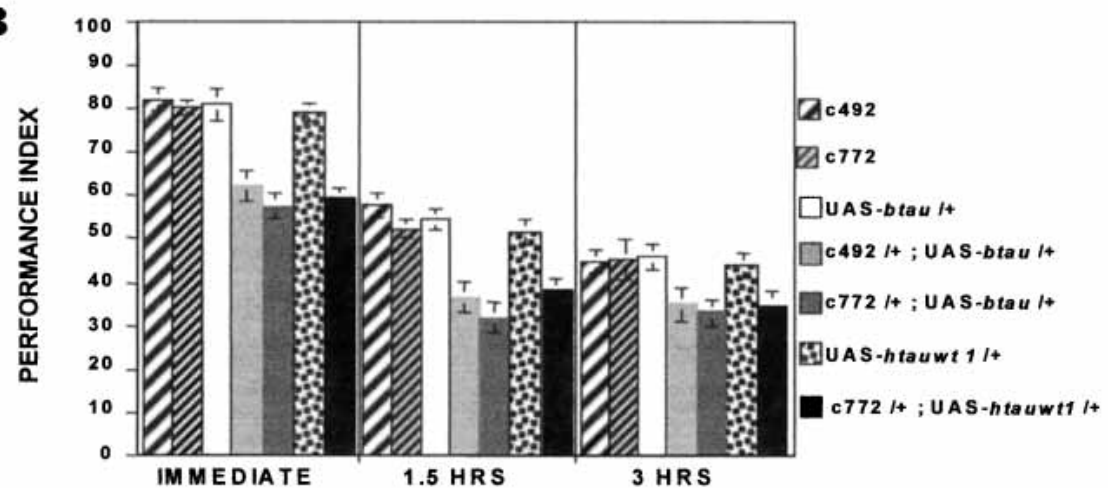

C

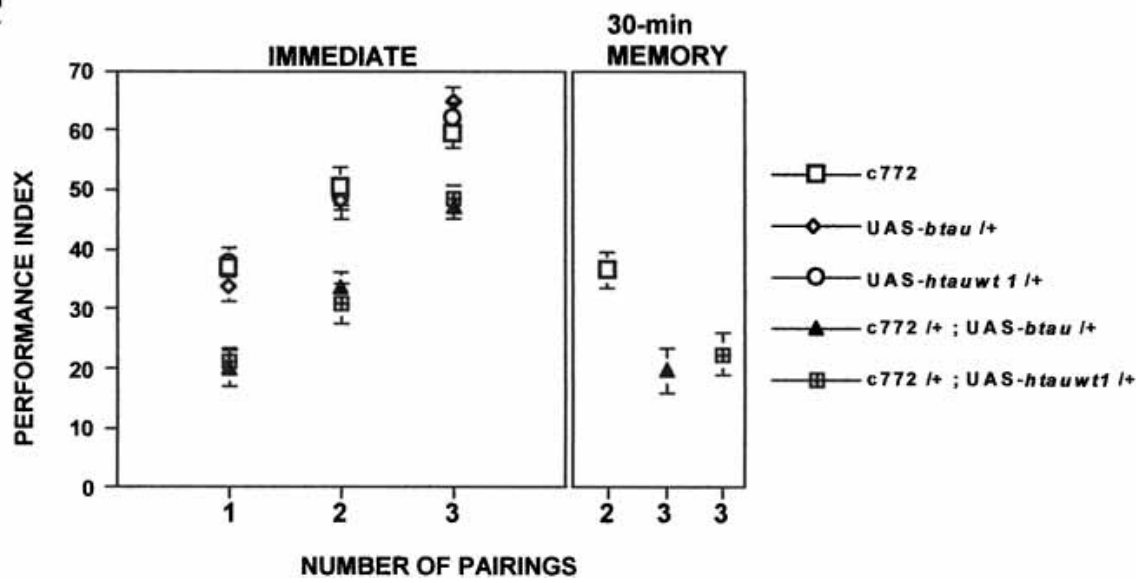

Figure 4 Experience-dependent nonassociative and associative behaviors in vertebrate tau-expressing animals and controls. ( $A$ ) Nonassociative pre-exposure effect. (1) Avoidance of benzaldehyde after pre-exposure to full-strength octanol and 90-V electric shock (filled bars) in comparison to avoidance without such pre-exposure (open bars; $n \geq 7)$. ANOVA revealed significant effects of treatment $\left(F_{(1,111)}=12.832, p<0.005\right)$, but not for genotype. (2) Avoidance of octanol after pre-exposure to benzaldehyde and 90-V electric shock (filled bars) in comparison to octanol avoidance without pre-exposure (open bars; $n \geq 7$ ). ANOVA revealed significant effects of treatment $\left(F_{(1,112)}=14.316, p<0.005\right)$, but not for genotype. (B) Olfactory memory after LONG paradigm conditioning. The mean Performance Index \pm SEM at the indicated times after conditioning for the genotypes indicated on the right of the graph are shown. Immediate (3-min) memory $(n \geq 9)$. For all other time intervals, $n \geq 8$. Two-way ANOVA revealed significant effects of genotype $\left[\left(F_{(6,72)}=9.765, p<0.005 ;\right.\right.$ immediate $\left.),\left(F_{(6,64)}=7.043, p<0.005 ; 1.5 \mathrm{~h}\right),\left(F_{(6,66)}=3.026, p<0.01 ; 3 \mathrm{~h}\right)\right]$ and time $\left(F_{(2,202)}=8.725\right.$, $p<0.005)$. Subsequent Dunnett's tests for each time interval did not reveal significant differences in performance among the $c 492 /+, c 772 /+$, UAS-btau/+, and UAS-htauwt1/+ control strains, or between the c492/+; UAS-btau/+, and c772/+; UAS-btau/+ and c772/+; UAS-htauwt1/+ heterozygotes. However, the differences between c492/+; UAS-btau/+ and c772/+; UAS-btau/+ heterozygotes and c772/+; UAS-htauwt1 and the control strains were highly significant $(p<0.001)$ for immediate memory and 1.5 -h memories. The differences among experimental and control strains at $3 \mathrm{~h}$ after training were not as significant $(p<0.05)$. Similar results were obtained with c492; UAS-htauwt1 heterozygotes (data not shown). (C) Performance after SHORT conditioning paradigm. The average performance (PI + SEM) of c772/+, UAS-btau/+ heterozygotes and UAS-htauwt $1 /+$ compared with that of c772/+; UAS-btau/+ and c772/+; UAS-htauwt 1/+ heterozygotes after one, two, and three CS/US pairings (number of pairings) is shown ( $n \geq 8$ ). The scale has been reduced for clarity. Two-way ANOVA indicated significant effects of genotype $\left[\left(F_{(2,125)}=12.665, p<0.005\right), 3\right.$-min memory (immediate), $\left(F_{(2,26)}=8.739, p<0.005, n \geq 7\right)$ for 30-min memory]. Subsequent Dunnett's tests revealed significant differences between the performance of $c 772 /+;$ UAS-btau/+ and c772/+; UAS-htauwt1/+ heterozygotes and control strains $(p<0.001$ for both) for each pairing. However, the performance of $c 772$; UAS-btau/+ and c772/+; UAS-htauwt1/+ heterozygotes after three pairings was not significantly different from that of controls after two pairings. Dunnett's tests for 30-min memory scores indicated that effects of genotype were attributable to depressed performance of c772; UAS-btau/+ and c772/+; UAS-htauwt1/+ heterozygotes in comparison to controls $(p<0.001)$. 
posure to the CS+ and the electric shock US is requisite for USdependent establishment of a CS+ versus CS - differential response (Joynes and Grau 1996), it is surprising that this experience-dependent nonassociative process was not affected by TAU accumulation (Figs. 4A and 5A). This may be because as Preat (1998) suggested, the CS+/US pre-exposure-dependent decline in subsequent response to the CS - is mediated at least in part by brain centers other than the MBs. Therefore, TAU accumulation under c772 and c492 in the various neurons outside the MBs did not affect this nonassociative experience-dependent response and is unlikely to underlie the observed learning and memory deficits.

Collectively, the results of the behavioral analyses indicate that the level of TAU within mushroom body neurons is essential for both olfactory learning elicited by each CS/US and memory retrieval or stability and this may underlie the cognitive deficits observed early in many human tauopathies. Because equivalent associative learning and memory decrements were observed irre-

\section{A}

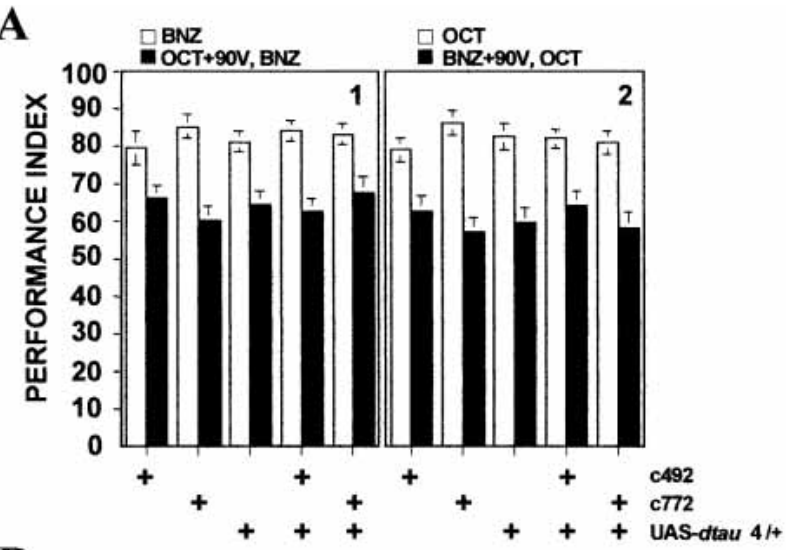

B
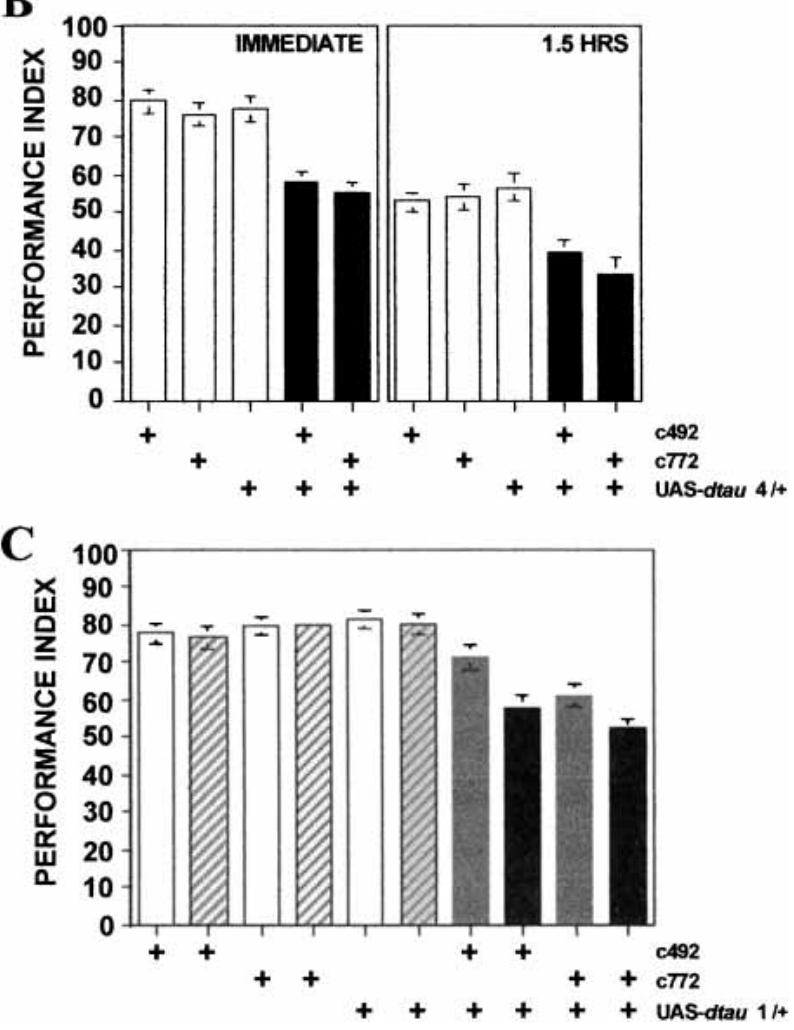

spective of whether vertebrate or Drosophila TAU accumulated in the MBs, potential conformational differences between dTAU and its vertebrate homologs were unlikely causal of these deficits. In contrast, the overall level of TAU within mushroom body neurons appeared to be of cardinal importance, as even a relatively acute elevation (Fig. 5C) of the protein yielded deficits. Moreover, the effects of TAU accumulation in the MBs were specific, because mere accumulation of non-Drosophila proteins, such as $\beta$-galactosidase (Skoulakis et al. 1993; Skoulakis and Davis 1996) and GAL4 (Figs. 4 and 5), or Drosophila proteins (Dubnau et al. 2001; McGuire et al. 2001) in these neurons, does not precipitate behavioral deficits. Interestingly, a decrease in short-term potentiation was uncovered in mice expressing a mutant allele of human $T A U$ in the hippocampus. In congruence with our results, TAU-dependent pathology appeared to develop much later than the deficits in short-term potentiation (Oddo et al. 2003). Moreover, in mice simultaneously carrying mutant tau, APP, and PS1 alleles, synaptic dysfunction and plasticity deficits preceded neurodegeneration (Oddo et al. 2003).

The robust decrements in associative learning and memory are consistent with the hypothesis that excess TAU binds to the neuronal microtubular cytoskeleton and this mediates the observed $\mathrm{MB}$ neuron dysfunction manifested as learning and memory deficits. Alternatively, the presence of excess TAU itself and not its association with the microtubular cytoskeleton is causal of the learning and memory deficits. How does elevation in TAU within MB neurons precipitate associative learning and memory deficits? First, inefficient vesicular traffic because of cytoskeletal function disruption may underlie the learning and

Figure 5 Experience-dependent nonassociative and associative behaviors in dtau-overexpressing animals and controls. (A) Nonassociative preexposure effect. (1) Avoidance of benzaldehyde after pre-exposure to full-strength octanol and $90-\mathrm{V}$ electric shock (filled bars) in comparison to avoidance without such pre-exposure (open bars; $n \geq 7$ ). ANOVA revealed significant effects of treatment $\left(F_{(1,78)}=13.784, p<0.005\right)$, but not for genotype, both in pre-exposed and non-pre-exposed animals. (2) Avoidance of octanol after pre-exposure to benzaldehyde and 90-V electric shock (filled bars) in comparison to octanol avoidance without preexposure (open bars; $n \geq 7$ ). ANOVA revealed significant effects of treatment $\left(F_{(1,84)}=14.026, p<0.005\right)$, but not for genotype, both in preexposed and non-pre-exposed animals. $(B)$ Olfactory memory after LONG paradigm conditioning. The mean Performance Index \pm SEM of c492/+, c772/+, UAS-dtau4/+ (open bars), and c492/+; UAS-dtau4/+ and c772/+; UAS-dtau4/+ (filled bars) is shown $(n \geq 9)$. Two-way ANOVA revealed significant effects of genotype $[(F, 52)=14.687, p<0.005 ; \mathrm{im}$ mediate 3-min); $\left.\left(F_{(4,49)}=9.327, p<0.005 ; 1.5 \mathrm{~h}\right)\right]$. Subsequent Dunnett's tests for each time interval did not reveal significant differences in performance among the c492/+, c772/+, UAS-dtau4/+ control strains or between the c492/+; UAS-dtau4/+ and c772; UAS-dtau4/+ heterozygotes. However, the differences between c492/+; UAS-dtau4/+ and c772; UAS-dtau4/+ heterozygotes and the control strains were highly significant $(p<0.001)$ for immediate memory and 1.5-h memories. (C) Performance of c492/+; UAS-dtau1/+ and c772; UAS-dtau1/+ heterozygotes with or without induction at $29^{\circ} \mathrm{C}$ after LONG conditioning. The average performance $(\mathrm{PI} \pm \mathrm{SEM})$ of animals raised at $23^{\circ}-24^{\circ} \mathrm{C}$ for control strains (c492/+, c772/+, UAS-dtau1/+) is indicated by open bars and c492/+; UAS-dtau1/+ and c772; UAS-dtau1/+ heterozygotes by gray filled bars. The performance of animals raised at $23^{\circ}-24^{\circ} \mathrm{C}$ and subsequently induced for $48-52 \mathrm{~h}$ at $29^{\circ} \mathrm{C}$ prior to behavioral experiments is indicated by the stippled bars for controls and the black-filled bars for c492/+; UASdtau $1 /+$ and $\mathrm{c772}$; UAS-dtau1/+ heterozygotes. Two-way ANOVA indicated significant effects of genotype $\left[\left(F_{(4,44)}=8.287, p<0.005\right)\right.$ for $23^{\circ}$ $24^{\circ} \mathrm{C}$ animals and $\left(F_{(4,48)}=10.016, p<0.005\right)$ for animals induced at $29^{\circ} \mathrm{C}$ ]. Subsequent Dunnett's tests revealed significant differences between the performances of c772; UAS-dtau1/+ heterozygotes and all control strains, as well as c492/+; UAS-dtau $1 /+$ strains when uninduced $(p<0.001)$. In contrast, both c772; UAS-dtau1/+ and c492/+; UASdtau $1 /+$ heterozygotes were different from controls when the animals were induced at $29^{\circ} \mathrm{C}$. 
memory deficits in animals accumulating TAU in their MBs. The highly ordered axonal microtubular cytoskeleton is thought to be essential for neuronal function as it is used for anterograde transport of synaptic vesicles (Baas 1999; Garcia and Cleveland 2001). Interestingly, anterograde axonal transport was inhibited in Drosophila larval motor axons accumulating bTAU (Torroja et al. 1999). Similarly, overexpression of TAU in cultured cell lines impaired kinesin-dependent transport of vesicles and organelles (Ebneth et al. 1998; Martin et al. 1999). Therefore, excess TAU could bind the axonal microtubular cytoskeleton and impair anterograde transport leading to decreased neurotransmitter pools in $\mathrm{MB}$ neurons. Consistent with this, immunoprecipitation from head lysates of animals accumulating bTAU in their MBs indicated that the vertebrate protein can, in fact, bind microtubules (E.M.C. Skoulakis, unpubl.). The apparent impairment of neurotransmission from MB neurons by abrogation of dynamindependent neurotransmitter reuptake affected memory retrieval, but not formation (Dubnau et al. 2001; McGuire et al. 2001). However, in contrast to this endocytotic blockade, TAU accumulation in the mushroom bodies affected both learning and memory stability or retrieval. If, indeed, TAU accumulation limits neurotransmitter availability by impairing anterograde transport, then the memory decrement of tau-expressing transgenics may underlie retrieval rather than memory stability deficits.

A more complex model indicates that excessive TAU bound to the microtubular cytoskeleton could impair intracellular traffic, but also interfere with the ability of other cellular proteins to interact with it as well. In fact, microtubular dynamics and interactive capacity have been reported to be significantly reduced in vitro, in ratios of greater than one molecule of TAU to 15 molecules of tubulin (Panda et al. 1999). This indicates that TAU overaccumulation had broader effects on MB physiology than blocking neurotransmission from these neurons. This is consistent with the learning deficits observed with TAU-accumulating animals and not with abrogation of neurotransmitter reuptake (Dubnau et al. 2001; McGuire et al. 2001). Excess TAU appears to interact with noncytoskeletal neuronal proteins because the adverse effects of TAU elevation are enhanced in flies coexpressing the $\beta$-amyloid-like (APPL) protein in Drosophila (Torroja et al. 1999). Moreover, the degenerative and synaptic dysfunction effects of TAU were enhanced in mice coexpressing APP (Lewis et al. 2001; Oddo et al. 2003).

Finally, the dynamic interaction of neuronal microtubules with microtubule-binding proteins (MAPs) has been proposed to play a role in learning and memory (Woolf et al. 1994, 1999). Furthermore, neuronal microtubules and their regulated interaction with associated MAPs have been proposed to act as neuonal computational elements (Mavromatos and Nanopoulos $1998 \mathrm{a}, \mathrm{b})$. The proper species and correct ratio of MAPs to microtubules are proposed to be essential for neuronal function in both models. An implicit prediction of these models is that excess TAU bound to the microtubular cytoskeleton would increase its stability and this perturbation in the stoichiometry would precipitate learning and memory dysfunction, which is consistent with our observations. These models also predict that increasing the level of other MAPs within the MBs is likely to have a similar effect on learning and memory as that observed for TAU, a hypothesis presently under investigation.

Although our data cannot distinguish among the models presented above, they strongly indicate that as for synaptic plasticity (Oddo et al. 2003), behavioral plasticity deficits are the earliest manifestation of tauopathies and precede overt neurodegeneration. The robust behavioral phenotype and the powerful genetic tools available in Drosophila can be readily used to investigate the nature of excess TAU-mediated learning and memory deficits.

\section{MATERIALS AND METHODS}

\section{Drosophila Culture and Strains}

Drosophila were cultured in standard cornmeal sugar food supplemented with soy flour and $\mathrm{CaCl}_{2}$ at $20^{\circ}-22^{\circ} \mathrm{C}$. The strains bearing transposons with tissue-restricted GAL4 expression (Brand and Perrimon 1993) in the mushroom bodies c772 (Yang et al. 1995) and c492 (Armstrong et al. 1998) were obtained from K. Kaiser (University of Glasgow, UK). Expression patterns were verified by crossing these GAL4 strains to UAS-lacZ reporter constructbearing animals (Brand and Perrimon 1993) and monitoring reporter gene activity in head cryosections (Skoulakis et al. 1993; Skoulakis and Davis 1996).

The bovine tau (UAS-btau) transgene-bearing strain was a kind gift of K. Ito (National Institute for Basic Biology, Japan; Ito et al. 1997), and the human tau transgene strain UAS-htauwt1 (Wittman et al. 2001) was obtained from Mel Feany (Harvard Medical School, Massachusetts). To generate transgenic animals with the Drosophila tau gene (dtau), the entire dtau cDNA was cloned (EcoRI/XhoI) into the pP[UAST] vector. Of the transgenic lines obtained, UAS-dtau 4 was selected for further work because of the intense eye color of homozygotes, indicating good expression of the transgene. Similarly, the UAS-dtau1 line was selected because it exhibited the weakest eye color, indicating low transgene expression. All other transformant lines exhibited intermediate eye colors. All strains were normalized to an isogenic $w^{1118}$ strain. To obtain flies for behavioral analyses, c772 and c492 homozygotes were crossed en masse to UAS-btau, UAS-htauwt1, UAS-dtau 4, and UAS-dtau1 homozygotes, and the progeny was collected and tested 3-5 d after emergence. Similarly, the UASbtau, UAS-htauwt1, UAS-dtau 4, and UAS-dtau1 homozygotes were crossed en masse to $w^{1118}$, to obtain heterozygotes used as controls. To elevate expression from the UAS-dtau1 transgene, flies raised at $23^{\circ}-24^{\circ} \mathrm{C}$ were subsequently placed at $29^{\circ} \mathrm{C}$ for $48-$ $52 \mathrm{~h}$ prior to behavioral experiments, or tissue isolation for RTPCR analysis.

To determine survival rates, groups of 40 animals of both sexes were collected within 2-4 d of emergence and were sequestered in food vials. The vials were changed weekly, and the percentage of survivors was determined from duplicate vials; the results of at least two independent experimental sets were averaged.

\section{Immunohistochemistry}

For immunohistochemistry or histology, 5- $\mu \mathrm{m}$ frontal paraffin sections of heads were obtained and processed as previously described (Skoulakis and Davis 1996; Crittenden et al. 1998). To determine the expression pattern under c492 and c772, an antibTAU monoclonal antibody (Sigma) was used at 1:1000 dilution. This antibody does not react with the Drosophila protein, as demonstrated by the lack of staining in control UAS-btau, c492, and c772 animals. The anti-LEO, anti-FASII, anti-HRP, and standard Hematoxylin-Eosin staining were used for neuroanatomical analyses as described previously (Crittenden et al. 1998).

\section{Western Blot Analysis}

Heads from c772, c492, c772; UAS-btau, and c492; UAS-btau animals were separated from bodies by vigorous shaking in liquid nitrogen and sieving. Heat-stable protein extracts were prepared essentially as in Heidary and Fortini (2001). The lysates were quantified with Bradford (Biorad) assays, Laemmli buffer was added, and the lysates were run on standard SDS-PAGE and blotted. The anti-bTAU monoclonal antibody (Sigma) was used at 1:1000. A polyclonal anti-TAU antibody (Dako) that identifies the human protein was used at 1:10,000. The results were visualized with enhanced chemiluminescence (Pierce).

\section{Reverse Transcription Polymerase Chain Reaction (RT-PCR)}

For RT-PCR, 40 heads were homogenized in $200 \mu \mathrm{L}$ of Trizol, and RNA was prepared as suggested by the manufacturer (GIBCO 
BRL). For Reverse Transcription (RT), $1 \mu \mathrm{g}$ of DNase I-treated total RNA was used per $50-\mu \mathrm{L}$ reaction along with twice the suggested amount of ImProm-II Reverse Transcriptase (Promega), and the reaction proceeded for $60 \mathrm{~min}$ at $42^{\circ} \mathrm{C}$. RT from transgenederived transcripts was achieved with a reverse primer (200 ng per reaction) specific for the unique SV40-derived sequence in pP[UAST] (Brand and Perrimon 1993) present at the 3 '-end of dtau transgenes (SV40A primer). Then $10 \%$ of each RT was subjected to 35 cycles PCR $\left(1 \mathrm{~min}\right.$ at $94^{\circ} \mathrm{C}, 45 \mathrm{sec}$ at $58^{\circ} \mathrm{C}, 2 \mathrm{~min}$ at $\left.72^{\circ} \mathrm{C}\right)$, using the SV40A and dtauF1 (5'-GATCGAGACCCTGAA GATG-3') primers for $d t a u$ RT products. As a qualitative control of the RT, $200 \mathrm{ng}$ of leo6.2 reverse primer was used, followed by PCR with leo-specific primers (Philip et al. 2001). Specificity of the reactions was tested with DNase I-treated, not reverse-trancribed, RNA.

\section{Behavioral Analyses}

Learning and memory were assessed using the negatively reinforced olfactory assay (Tully and Quinn 1985), coupling aversive odors as conditioned stimuli (CS+ and CS -), and electric shock as the unconditioned stimulus (US). LONG TRAINING was performed using previously described assay modifications (Skoulakis and Davis 1996; Philip et al. 2001). The SHORT conditioning assay was performed as described previously (Beck et al. 2000). Because the earliest possible time that we can test the animals past the CS+ and US presentation is 180-200 sec, our measurements cannot differentiate between "acquisition" and "3-minute memory." This earliest performance assessment is referred to as "learning." Sensory control experiments were performed as previously described (Skoulakis and Davis 1996; Philip et al. 2001).

For CS+/US pre-exposure experiments (Preat 1998), flies were given $60 \mathrm{sec}$ of benzaldehyde, concomitant with $1190-\mathrm{V}$ electric shocks. Subsequently, their avoidance of octanol versus air was quantified. Complementary experiments pre-exposing flies to octanol and $1190-\mathrm{V}$ electric shocks and evaluating benzaldehyde avoidance were performed in parallel.

Olfactory trap assays with the attractive odorant geraniol were performed as described in Phillip et al. (2001). Briefly, 10 male flies were placed in a $100 \times 15$-mm plastic Petri dish containing moistened Whatman paper, and the olfactory trap was made by cutting the bottoms of a $0.5-\mathrm{mL}$ and a $1.5-\mathrm{mL}$ Eppendorf tube and attaching them via their cut bottoms. Then $200 \mu \mathrm{L}$ of $1 \%$ agarose containing $0.05 \%$ geraniol was placed in the lid of the $1.5-\mathrm{mL}$ Eppendorf tube, which was subsequently tightly closed. Attraction to the odor was assessed at $23^{\circ}-24^{\circ} \mathrm{C}$ in the dark.

\section{Statistical Analysis}

Untransformed (raw) data were analyzed parametrically with the JMP3.1 statistical software package (SAS Institute Inc.) as described (Skoulakis et al. 1993; Skoulakis and Davis 1996). To maintain a constant experimentwise error rate, the significance level was adjusted as suggested by Sokal and Rolf (1981). Following initial ANOVA, planned multiple comparisons, comparisons to a control strain (Dunnett's test), or Tukey-Kramer tests were performed as indicated in the figure legends.

\section{ACKNOWLEDGMENTS}

The authors are indebted to Rita Williamson for invaluable help with husbandry and propagation of strains. We thank Mary McCrady and Gabriel Ayala for technical assistance and Summer F. Acevedo for help with stocks and valuable discussions. We thank K. Kaiser, Mel Feany, and the Bloomington stock center for fly stocks and G. Heidary and M. Fortini for cDNA and antibody to Drosophila TAU protein. This work was supported by an Interdisciplinary Research Initiative Grant (E.M.C.S. and D.V.N.) from Texas A\&M University.

The publication costs of this article were defrayed in part by payment of page charges. This article must therefore be hereby marked "advertisement" in accordance with 18 USC section 1734 solely to indicate this fact.

\section{REFERENCES}

Armstrong, J.D., deBelle, J.S., Wang, Z., and Kaiser, K. 1998. Metamorphosis of the mushroom bodies; large scale rearrangements of the neural substrates for associative learning and memory in Drosophila. Learn. Mem. 5: 102-114.

Baas, P.W. 1999. Microtubules and neuronal polarity: Lessons from mitosis. Neuron 22: 23-31.

Bancher, C., Brunner, C., Lassmann, H., Budka, H., Jellinger, K., Wiche, G., Seitelberger, F., Grundke-Iqbal, I., and Wisniewski, H.M. 1989. Accumulation of abnormally phosphorylated tau precedes the formation of neurofibrillary tangles in Alzheimer's disease. Brain Res. 477: 90-99.

Beck, C.D.O., Schroeder, B., and Davis, R.L. 2000. Learning performance of normal and mutant Drosophila after repeated conditioning trials with discrete stimuli. J. Neurosci. 20: 2944-2953.

Braak, E., Braak, H., and Mandelkow, E.-M. 1994. A sequence of cytoskeleton changes related to the formation of neurofibrillary tangles and neuropil threads. Acta Neuropathol. 87: 554-567.

Brand, A.H. and Perrimon, N. 1993. Targeted gene expression as a means of altering cell fates and generating dominant phenotypes. Development 118: 401-415.

Brion, J.P., Tremp, G., and Octave, N.J. 1999. Transgenic expression of the shortest human tau affects its compartmentalization and its phosphorylation as in the pretangle state of Alzheimer's disease. Am. J. Pathol. 154: 255-270.

Buchanan, R.L. and Benzer, S. 1993. Defective glia in the Drosophila brain degeneration mutant drop-dead. Neuron 10: 839-850.

Buee, L., Bussiere, T., Buee-Scherrer, V., Delacourte, A., and Hof, P.R. 2000. Tau protein isoforms, phosphorylation and role in neurodegenerative disorders. Brain Res. Rev. 33: 95-130.

Cheng, Y., Endo, K., Wu, K., Rodan, A.R., Heberlein, U., and Davis, R.L. 2001. Drosophila fasciclin II is required for the formation of odor memories and for normal sensitivity to alcohol. Cell 105: 757-768.

Connoly, J.B., Roberts, I.J.H., Armstrong, J.D., Kaise, K., Forte, M., Tully, T., and O'Kane, C.J. 1996. Associative learning disrupted by impaired Gs signaling in Drosophila mushroom bodies. Science 274: $2104-2107$.

Crittenden, J.R., Skoulakis, E.M.C., Han, K.-A., Kalderon, D., and Davis, R.L. 1998. Tripartite mushroom body architecture revealed by antigenic markers. Learn. Mem. 5: 38-51.

DeBelle, S.J. and Heisenberg, M. 1994. Associative odor learning in Drosophila is abolished by chemical ablation of mushroom bodies. Science 263: 692-695.

Dubnau, J., Grady, L., Kitamoto, T., and Tully, T. 2001. Disruption of neurotransmission in Drosophila mushroom body blocks retrieval, but not acquisition of memory. Nature 411: 476-480.

Duffy, J.B. 2002. GAL4 system in Drosophila: A fly geneticist's Swiss army knife. Genesis 34: 1-15.

Ebneth, A., Godemann, R., Stamer, K., Illenberger, S., Trinczeck, B. Mandelkow, E.-M., and Mandelkow, E. 1998. Overexpression of Tau protein inhibits Kinesin-dependent trafficking of vesicles, mitochondria and endoplasmic reticulum: Implications for Alzheimer's disease. J. Cell Biol. 143: 777-794.

Garcia, M.L. and Cleveland, D.W. 2001. Going new places using an old MAP: tau, microtubules and human neurodegenerative disease. Curr. Opin. Cell Biol. 13: 41-48.

Heidary, G. and Fortini, M. 2001. Identification and characterization of the Drosophila tau homolog. Mech. Dev. 108: 171-178.

Himmler, A. 1989. Structure of the bovine Tau gene: Alternatively spliced transcripts generate a protein family. Mol. Cell. Biol. 9: 1389-1396.

Himmler, A., Drechsel, D., Kirschner, M.W., and Martin, J.D.W. 1989. Tau consists of a set of proteins with repeated C-terminal microtubule-binding domains and variable N-terminal domains. $\mathrm{Mol}$. Cell. Biol. 9: 1381-1388.

Hutton, M., Lewis, J., Dickson, D., Yen, S.-H., and McGowan, E. 2001. Analysis of tauopathies with transgenic mice. Trends Mol. Med. 7: 467-470.

Ito, K., Sass, H., Urban, J., Hofbauer, A., and Schneuwly, S. 1997. GAL4-responsive UAS-tau as a tool for studying the anatomy and development of the Drosophila central nervous system. Cell Tissue Res. 290: 1-10.

Jackson, G.R., Wiedau-Pazos, M., Wagle, N., Brown, C.A., Massachi, S., and Geschwind, D.H. 2002. Human wild-type tau interacts with wingless pathway components and produces neurofibrillary pathology in Drosophila. Neuron 34: 509-519.

Joynes, R.L. and Grau, J.W. 1996. Mechanisms of Pavlovian conditioning: Role of protection form habituation in spinal conditioning. Behav. Neurosci. 110: 1375-1387.

Lee, V.M.-Y., Goedert, M., and Trojanowski, J.Q. 2001. Neurodegenerative tauopathies. Annu. Rev. Neurosci. 24: 1121-1159. 
Lewis, J., McGowan, E., Rockwood, J., Melrose, H., Nacharaju, P., Van Slegtenhorst, M., Gwinn-Hardy, K., Paul Murphy, M., Baker, M., Yu X., et al. 2000. Neurofibrillary tangles, amyotrophy and progressive motor disturbance in mice expressing mutant (P301L) tau protein. Nat. Genet. 1: 127 .

Lewis, J., Dickson, D.W., Lin, W.L., Chisholm, L., Corral, A., Jones, G., Yen, S.H., Sahara, N., Skipper, L., Yager, D., et al. 2001. Enhanced neurofibrillary degeneration in transgenic mice expressing mutant TAU and APP. Science 293: 1446-1447.

Martin, M., Iyadurai, S.J., Gassman, A., Gindhart, J.G.J., Hays, T.S., and Saxton, W.M. 1999. Cytoplasmic dynein, the dynactin complex, and kinesin are interdependent and essential for fast axonal transport. Mol. Biol. Cell 10: 3717-3728.

Mavromatos, N.E. and Nanopoulos, D.V. 1998a. On quantum mechanical aspects of microtubules. Int. J. Mod. Phys. B12: 517-542. - 1998b. Quantum mechanics in cell microtubules: Wild imagination or realistic possibility? Advances Struct. Biol. 5: 283-318.

McGuire, S.E., Le, P.T., and Davis, R.L. 2001. The role of Drosophila mushroom body signaling in olfactory memory. Science 293: 1330-1333.

Mitchison, T. and Kirschner, M.W. 1984. Dynamic instability of microtubule growth. Nature 312: 237-242.

Oddo, S., Caccamo, A., Shepherd, J.D., Murphy, M.P., Golde, T.E., Kayed, R., Metherate, R., Mattson, M.P., Akbari, Y., and LaFerla, F.M. 2003. Triple-transgenic model of Alzheimer's disease with plaques and tangles: Intracellular $\mathrm{Ab}$ and synaptic dysfunction. Neuron 39: 409-421.

Panda, D., Miller, H.P., and Wilson, L. 1999. Rapid treadmilling of brain microtubules free of microtubule-associated proteins in vitro and its suppression by tau. Proc. Natl. Acad. Sci. 96: 12459-12464.

Philip, N., Acevedo, S., and Skoulakis, E.M.C. 2001. Conditional rescue of olfactory learning and memory defects in mutants of the $14-3-3 z$ gene leonardo. J. Neurosci. 21: 8417-8425.

Preat, T. 1998. Decreased odor avoidance after electric shock in Drosophila mutants biases learning and memory tests. J. Neurosci.
18: $8534-8538$.

Roman, G. and Davis, R.L. 2001. Molecular biology and anatomy of Drosophila olfactory associative learning. BioEssays 23: 571-581.

Skoulakis, E.M.C. and Davis, R.L. 1996. Olfactory learning deficits in mutants for leonardo, a Drosophila gene encoding a 14-3-3 protein. Neuron 17: 931-944.

Skoulakis, E.M.C., Kalderon, D., and Davis, R.L. 1993. Preferential expression in mushroom bodies of the catalytic subunit of protein kinase A and its role in learning and memory. Neuron 11: 197-208

Sokal, R.R. and Rohlf, F.J. 1981. Biometry: The principles and practice of statistics in biological research, 2nd ed. W.H. Freeman, New York.

Torroja, L., Hsin, C., Kotovsky, I., and White, K. 1999. Neuronal overexpression of APPL, the Drosophila homologue of the amyloid precursor protein (APP), disrupts axonal transport. Curr. Biol. 9: 489-492.

Tully, T. and Quinn, W. 1985. Classical conditioning and retention in normal and mutant Drosophila melanogaster. J. Comp. Physiol. 157: $263-277$.

Wittman, C.W., Wszolek, M.F., Shulman, J.M., Salvaterra, P.M., Lewis, J., Hutton, M., and Feany, M.B. 2001. Tauopathy in Drosophila: Neurodegeneration without neurofibrillary tangles. Science 293: $711-714$

Woolf, N.J., Young, S.L., Johnson, G.V.W., and Fanselow, M.S. 1994 Pavlovian conditioning alters cortical microtubule-associated protein-2. NeuroReport 5: 1045-1048.

Woolf, N.J., Zinnerman, M.D., and Johnson, G.V. 1999. Hippocampal microtubule-associated protein-2 alterations with contextual memory. Brain Res. 821: 241-249.

Yang, M.Y., Armstrong, J.D., Volisky, I., Strausfeld, N.J., and Kiser, K. 1995. Subdivision of the Drosophila mushroom bodies by enhancer-trap expression patterns. Neuron 15: 45-54.

Received September 12, 2003; accepted in revised form January 6, 2004. 


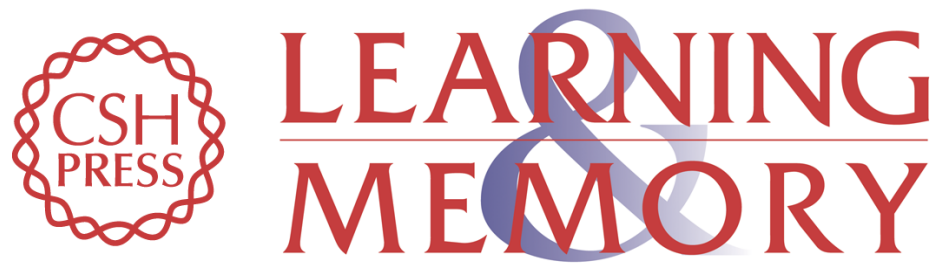

\section{Learning and Memory Deficits Upon TAU Accumulation in Drosophila Mushroom Body Neurons}

Andreas Mershin, Elias Pavlopoulos, Olivia Fitch, et al.

Learn. Mem. 2004, 11:

Access the most recent version at doi:10.1101/lm.70804

References This article cites 43 articles, 16 of which can be accessed free at:

http://learnmem.cshlp.org/content/11/3/277.full.html\#ref-list-1

License

Email Alerting Receive free email alerts when new articles cite this article - sign up in the box at the Service top right corner of the article or click here. 